\title{
Predicting phenotypes of asthma and eczema with machine learning
}

\author{
Mattia CF Prosperi ${ }^{1,2^{*}}$, Susana Marinho ${ }^{2}$, Angela Simpson ${ }^{2}$, Adnan Custovic $^{2 \dagger}$, lain E Buchan ${ }^{1+}$ \\ From The 3rd Annual Translational Bioinformatics Conference (TBC/ISCB-Asia 2013) \\ Seoul, Korea. 2-4 October 2013
}

\begin{abstract}
Background: There is increasing recognition that asthma and eczema are heterogeneous diseases. We investigated the predictive ability of a spectrum of machine learning methods to disambiguate clinical sub-groups of asthma, wheeze and eczema, using a large heterogeneous set of attributes in an unselected population. The aim was to identify to what extent such heterogeneous information can be combined to reveal specific clinical manifestations.
\end{abstract}

Methods: The study population comprised a cross-sectional sample of adults, and included representatives of the general population enriched by subjects with asthma. Linear and non-linear machine learning methods, from logistic regression to random forests, were fit on a large attribute set including demographic, clinical and laboratory features, genetic profiles and environmental exposures. Outcome of interest were asthma, wheeze and eczema encoded by different operational definitions. Model validation was performed via bootstrapping.

Results: The study population included 554 adults, 42\% male, 38\% previous or current smokers. Proportion of asthma, wheeze, and eczema diagnoses was 16.7\%, 12.3\%, and 21.7\%, respectively. Models were fit on 223 nongenetic variables plus 215 single nucleotide polymorphisms. In general, non-linear models achieved higher sensitivity and specificity than other methods, especially for asthma and wheeze, less for eczema, with areas under receiver operating characteristic curve of $84 \%, 76 \%$ and $64 \%$, respectively. Our findings confirm that allergen sensitisation and lung function characterise asthma better in combination than separately. The predictive ability of genetic markers alone is limited. For eczema, new predictors such as bio-impedance were discovered.

Conclusions: More usefully-complex modelling is the key to a better understanding of disease mechanisms and personalised healthcare: further advances are likely with the incorporation of more factors/attributes and longitudinal measures.

\section{Background}

Asthma is the most common chronic disease in developed countries, however, the drug armamentarium available to manage the condition is modest[1]. There is increasing recognition that asthma is a heterogeneous disease with multiple endotypes, which may have similar clinical manifestations, or phenotypes, but different underlying pathophysiological causes[2,3]. Appropriate

\footnotetext{
* Correspondence: mattia.prosperi@manchester.ac.uk

+ Contributed equally

${ }^{1}$ Centre for Health Informatics, Institute of Population Health, Faculty of Medical and Human Sciences, University of Manchester, Manchester, UK Full list of author information is available at the end of the article
}

identification of such endotypes is critical for the understanding of the disease mechanism and the development of personalised approaches to its management[4]. Sensitisation to allergens from several sources (such as pets, dust mites, cockroaches, and pollens) has been independently associated with asthma and asthma-related symptoms[5-8], and among asthmatic patients with the severity of the disease[9-12]. Also, sensitisation to inhalant allergens has been found to be associated with diminished lung function and increased airway responsiveness[13].

It remains unclear to what extent allergen sensitisation and lung function markers (e.g., airway reactivity, airway 
inflammation), in conjunction with a broader set of other potentially relevant information (e.g. environmental exposures or genetic characteristics), contribute towards specific clinical manifestations of different atopic diseases (e.g. asthma $v s$. eczema). In the past decades, several approaches to predict such current or subsequent clinical manifestations, both in children and adults, have been introduced[14-22]. The performance of prediction models varies in relation to different population strata, and obviously in relation to the clinical outcome or end-point definitions. For instance, one of the earliest works, by Castro-Rodríguez et al.[14], devised a rule-based asthma predictive index to predict subsequent asthma amongst young children with a history of wheezing, attaining sensitivity $\sim 0.4$ at $\sim 0.8$ specificity on various time points. The recent work by Chatzimichail et al.[23] reported $\sim 0.95$ of both sensitivity and specificity in predicting current asthma in symptomatic preschool children, using a machine learning approach based on previous symptoms, medications, allergen sensitisation and lung function.

In this work, using a rich data set from an unselected cross-sectional population study, different operational definitions of current asthma, wheeze and eczema are carefully derived, and we analyse their prognostic factors from a large set of markers, which includes demographic, clinical, laboratory features, genetic profiles and environmental exposures. Of note, previous diagnoses (along with anti-asthma medication usage) are removed on purpose from the input set, as many clinical outcome definitions are recursively based on them. The aim is to identify to which extent such heterogeneous information contributes and combines towards the prediction of a specific clinical presentation - comparing linear and non-linear machine learning models fitted with different feature combinations - and eventually prepare the grounds for the deployment of a personalized diagnostic tool.

\section{Methods}

The study population comprised a cross-sectional sample of adult individuals, age $\geq 18$ years, including representatives of the general population enriched by subjects with asthma $[13,24]$. For the sample from the general population, we approached parents of children who have been under active follow-up in the Manchester Asthma and Allergy Study (population-based birth cohort study)[25]. The population of subjects with asthma included well-phenotyped adults who were identified from a clinical trials database, and had both a history of physician-diagnosed asthma and asthma symptoms within the previous 12 months[26,27]. The study was approved by the Local Research Ethics Committee (05/Q1406/70) and is registered as N0226171141. Written informed consent was obtained from all subjects.

A total of 1,102 attributes of the study participants were collected across a large heterogeneous information spectrum, including interviewer-administered questionnaires, laboratory measurements, doctors' diagnoses and environmental exposures. The collected data included:

- demographic information (e.g. gender, ethnicity, age, place of residence);

- questionnaire data related to symptom presence and severity (e.g. wheeze, shortness of breath, chronic cough), previous/current diagnoses of asthma, hay fever, eczema, food allergies or other illnesses;

- use of anti-asthma medications (e.g. short-acting beta agonists [SABA], long-acting beta agonists [LABA], inhaled corticosteroids [ICS]);

- questionnaire data on smoking and alcohol drinking habits, current pet ownership, indoor environmental conditions (e.g. rugs, beds, type of house heating, latex usage), occupation and occupation-related accidents;

- objective measures on environmental exposure to house dust mite (Der p 1), cat (Fel d 1) and dog (Can f 1) allergen determined in dust samples collected from homes using enzyme-linked immunosorbent assays (ELISAs);

- objective measures on environmental exposure to endotoxin (marker of exposure to gram-negative bacteria) and beta glucan (marker of exposure to moulds) determined in dust samples collected from homes;

- body measurements (e.g. height, weight, body mass index [BMI], fat percentage, whole body impedance); - lung function measurements (e.g. forced expiratory volume in 1 second $\left[\mathrm{FEV}_{1}\right]$, forced vital capacity [FVC], peak expiratory flow [PEF], functional reserve capacity [FRC] and residual volume [RV], total lung capacity [TLC], forced expiratory flow $25-75 \%\left[\mathrm{FEF}_{25-75}\right]$ and specific airway resistance [sRaw]);

- measurement of airway inflammation (exhaled nitric oxide $[\mathrm{eNO}]$ )

- measurement of airway hyper-responsiveness using methacholine challenge, expressed as a provocative concentration of methacholine needed to produce a $20 \%$ fall in $\mathrm{FEV}_{1}$ (PC20), and methacholine doseresponse slope (MDRS);

- assessment of atopic status using (i) skin prick tests (SPT), (ii) measurement of serum allergen-specific Immunoglobulin E values (IgE), and (iii) component resolved diagnostics using an immuno-dot blot as previously described[28].

In addition, as part of a candidate gene association study, subjects were genotyped for 215 single nucleotide polymorphisms (SNPs) in genes found to be associated with asthma in previous studies (including polymorphisms in chromosomal regions 20p13-p12 and 17q12-21)[24].

We used the following (partly overlapping) definitions of asthma, wheeze and eczema. 
Asthma was encoded with three "operational definitions" determined by questionnaire, specifically:

i. current asthma (CA), based on De Marco et al.[29], defined as asthma ever confirmed by a doctor and at least one symptom of wheeze, nocturnal chest tightness, asthma attack within the past 12 months, attacks of breathlessness following activity, at rest or at night-time, having taken anti-asthma medication; ii. level-2 ECRHS II[30] definition (A2), as two positive answers to the questions "have you been woken by an attack of shortness of breath at any time in the last 12 months", "have you had an attack of asthma in the last 12 months", "are you currently taking any medicines including inhalers, aerosol or tablets for asthma";

iii. level-3 ECRHS II definition (A3), as three positive answers out of the set described at the previous point.

Current wheeze $(\mathrm{CW})$ was defined, according to Pekkanen et al.[31], as the presence of wheeze/breathlessness in the previous 12 months outside colds.

Eczema was defined as self-diagnosed (SDE) or doctorconfirmed (DDE) eczema.

Out of the 1,102 original non-genetic attributes, 223 were selected by clinical researchers, excluding factors considered as irrelevant or completely redundant, and those that were defining features of diagnoses. Attributes were grouped into: demographic/environmental variables ( $\mathrm{n}=74$, including age, gender, $\mathrm{BMI}$, whole body impedance, housing conditions, pet ownership, plus $\mathrm{n}=56$ variables measuring environmental exposures to endotoxin, beta glucan and indoor allergens); lung function, airway inflammation and airway hyperresponsiveness markers $(\mathrm{n}=12$, including eNO, \% predicted $\mathrm{FEV}_{1}$, FVC, $\mathrm{FEV}_{1} / \mathrm{FVC}, \mathrm{FEF}_{25-75}$, sRaw, PEF, TLC, $\mathrm{RV}$, methacholine challenge MDRS and PC20); allergen sensitization assessed either by skin prick testing, specific serum IgE measurement or component resolved diagnostics $(\mathrm{n}=8, \mathrm{n}=7, \mathrm{n}=66$, respectively), recording mean wheal diameters (MWD) and IgE levels, which were either log-transformed or discretized into ordered quartile categories (where a negative or below limit of detection result was the zero-order category). All 215 SNPs were retained and merged to the data set. Before data merge, raw SNP data were processed through linkage-disequilibrium filtering/imputation using Haploview [32] and the method of Gabriel et al.[33] (as described in the previous work by Marinho et al.[24]). Other missing values were replaced by column-wise median and modes depending on the data types.

For descriptive statistics and comparison with other prediction methods, information about previous diagnoses and medication usage (ICS, SABA, LABA) was retained but not used as input for the main models.
Main-effects logistic regression (LR) models were fitted selecting features by means of the LogitBoost algorithm [34]. For comparison purposes, a LR model made by the best single predictor according to the Akaike information criterion (named one rule, OR) was considered[35]. A decision tree model (DT)[36] and a decision tree ensemble, the random forest (RF, 250 trees)[37] were also evaluated, along with the AdaBoost (AB) classifier[38]. Goodness-offit functions examined were: accuracy, i.e. percentage of correctly classified cases; area under the receiver operating characteristic curve (AUROC), which is equal to the probability that a classifier ranks a randomly chosen positive instance (e.g. condition present/diagnosed) higher than a randomly chosen negative one (e.g. condition absent); sensitivity, i.e. the probability that the classification is positive when the condition is present (true positive rate); specificity, i.e. the probability that the classification is negative when the condition is not present (true negative rate). Model performance was estimated and compared as extrasample via bootstrapping (100 replicates), considering outof-bag distributions, and assessing significance via t-tests adjusted for sample overlap and multiple comparisons [39-41]. Attribute importance was assessed by means of $\mathrm{RF}$, calculating the average re-scaled (i.e. divided by its standard error) decrease in accuracy by variable randomization (repeated for 1000 times), and comparing it against a null distribution obtained by shuffling outcome labels, calculating p-values according to the method of Altmann et al.[42] and previous works[43,44]. All analyses were carried out using R software (http://www.r-project.org/).

\section{Results}

\section{Characteristics of the study population}

The study population included 554 subjects, with a mean (standard deviation) age of 43 (5) years at the time of the assessment, $42 \%$ male, $38 \%$ previous or current smokers. The proportion of CA, CW and DDE were $16.7 \%, 12.3 \%$, and $21.7 \%$, respectively. Subjects' characteristics are described in detail in Table 1, as well as cross-tabulation of outcomes. There was a high level of agreement between SDE and DDE (95.5\%), as well as between CA, A2, A3 and CW (from 95.3\% of CA vs. A2 to $87.7 \%$ of CA vs. A3), as expected by their intersecting definitions. The lowest agreement was found between SDE and CW (73.4\%). For ease of reading, we have omitted information about genetic data, which has been described in detail previously[24] (available upon request). Of note, $73.2 \%$ attributes had no missing data, and the amount of missingness in the rest was a median (interquartile range, IQR) of $0.7 \%(0.2 \%-2.2 \%)$.

\section{Model inference}

Given the levels of agreement between outcomes, inference results will be presented here for $\mathrm{CA}, \mathrm{CW}$ and $\mathrm{DDE}$ 
Table 1 Study data

\begin{tabular}{|c|c|c|c|}
\hline \multirow{2}{*}{\multicolumn{2}{|c|}{ 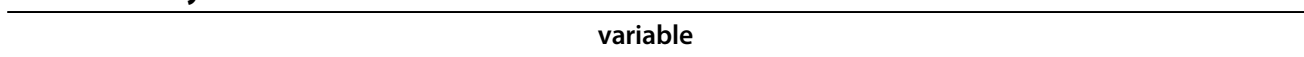 }} & \multirow{3}{*}{$\begin{array}{l}\text { median (IQR) } \\
42.6(39.7-45.7)\end{array}$} & \multirow{3}{*}{$\frac{\text { \#missing (\%) }}{0(0 \%)}$} \\
\hline & & & \\
\hline age (years) & & & \\
\hline \multicolumn{2}{|l|}{ year of birth } & $1964(1961-1967)$ & $0(0 \%)$ \\
\hline \multicolumn{2}{|l|}{ body mass index (BMI) } & $26(23.6-29.1)$ & $2(0.4 \%)$ \\
\hline \multicolumn{2}{|l|}{ whole body impedance } & $613.5(550-685)$ & $34(6.1 \%)$ \\
\hline \multicolumn{2}{|l|}{ fat $\%$} & $29.5(23.7-36)$ & $34(6.1 \%)$ \\
\hline \multicolumn{2}{|l|}{ exhaled nitric oxide (eNO), ppb ( $\log _{\mathrm{e}}$ scale) } & $2.8(2.4-3.3)$ & $94(17.0 \%)$ \\
\hline \multicolumn{2}{|l|}{ specific airway resistance (sRaw), kPa/s (loge scale) } & $-0.1(-0.3-0.1)$ & $11(2.0 \%)$ \\
\hline \multicolumn{2}{|l|}{ peak expiratory flow (PEF) \% predicted } & $\begin{array}{l}113.1(102.1- \\
124.6)\end{array}$ & $9(1.6 \%)$ \\
\hline \multicolumn{2}{|l|}{ forced vital capacity (FVC) \% predicted } & $\begin{array}{l}114.5(105.6- \\
123.1)\end{array}$ & $11(2.0 \%)$ \\
\hline \multicolumn{2}{|c|}{ forced expiratory volume in 1 second (FEV^ \% predicted } & $106(98.6-115.5)$ & $10(1.8 \%)$ \\
\hline \multicolumn{2}{|l|}{ forced expiratory flow $\left(\mathrm{FEF}_{25-75}\right) \%$ predicted } & $80(66-96.3)$ & $11(2.0 \%)$ \\
\hline \multicolumn{2}{|l|}{ total lung capacity (TLC) } & $108.5(101-116.9)$ & $11(2.0 \%)$ \\
\hline \multicolumn{2}{|l|}{ residual volume (RV) } & $113(98.6-127.8)$ & $11(2.0 \%)$ \\
\hline \multicolumn{2}{|l|}{ FEVj/FVC ratio } & $0.8(0.8-0.8)$ & $11(2.0 \%)$ \\
\hline \multicolumn{2}{|c|}{$\begin{array}{l}\text { provocative concentration of methacholine needed to produce a } 20 \% \text { fall in FEVj (PC20), of those completing the } \\
\text { test }\end{array}$} & $5.3(1.2-9.0)$ & $43(7.8 \%)$ \\
\hline \multicolumn{2}{|c|}{ methacholine dose-response slope (MDRS), transformed as 100/(MdRS+10) } & $5.7(4.2-7.5)$ & $43(7.8 \%)$ \\
\hline \multicolumn{2}{|c|}{ variable } & $\mathrm{N}(\%)$ & \#missing (\%) \\
\hline Gender & male & $234(42.2 \%)$ & $0(0 \%)$ \\
\hline \multirow[t]{3}{*}{ smoking status } & never & $341(61.6 \%)$ & $0(0 \%)$ \\
\hline & ex-smoker & $144(26 \%)$ & $0(0 \%)$ \\
\hline & current & $69(12.5 \%)$ & $0(0 \%)$ \\
\hline \multicolumn{2}{|l|}{ cat/dog ownership } & $186(33.6 \%)$ & $1(0.2 \%)$ \\
\hline \multirow[t]{7}{*}{ allergen sensitisation by skin prick test (SPT) } & dust mite (mean wheal diameter $>3 \mathrm{~mm}$ ) & $162(29.3 \%)$ & $1(0.2 \%)$ \\
\hline & cat (mean wheal diameter $>3 \mathrm{~mm}$ ) & $106(19.2 \%)$ & $1(0.2 \%)$ \\
\hline & dog (mean wheal diameter $>3$ mm) & $48(8.6 \%)$ & $1(0.2 \%)$ \\
\hline & tree (mean wheal diameter $>3 \mathrm{~mm}$ ) & $76(13.8 \%)$ & $1(0.2 \%)$ \\
\hline & grass (mean wheal diameter $>3 \mathrm{~mm}$ ) & $129(23.4 \%)$ & $1(0.2 \%)$ \\
\hline & mould (mean wheal diameter $>3$ mm) & $16(2.9 \%)$ & $1(0.2 \%)$ \\
\hline & peanut (mean wheal diameter >3 mm) & $9(1.7 \%)$ & $1(0.2 \%)$ \\
\hline \multicolumn{2}{|l|}{ bird ownership } & $13(2.4 \%)$ & $1(0.2 \%)$ \\
\hline \multirow[t]{2}{*}{ medications in the past three months } & short-acting beta agonists (SABA) & $34(6.1 \%)$ & $1(0.2 \%)$ \\
\hline & $\begin{array}{l}\text { inhaled corticosteroids (ICS) or ICS/long-acting beta agonists } \\
\text { (LABA) }\end{array}$ & $37(6.7 \%)$ & $1(0.2 \%)$ \\
\hline illness or problem caused by eating a particu & food or foods, ever & $97(17.5 \%)$ & $1(0.2 \%)$ \\
\hline accident at home, work or elsewhere exposir & to high levels of vapours, gas or dust & $22(4 \%)$ & $2(0.4 \%)$ \\
\hline carpets in the house & & $292(52.8 \%)$ & $1(0.2 \%)$ \\
\hline gas stove in the house & & $432(78.1 \%)$ & $1(0.2 \%)$ \\
\hline electric stove in the house & & $231(41.8 \%)$ & $1(0.2 \%)$ \\
\hline job causing wheezing problems & & $33(6 \%)$ & $3(0.5 \%)$ \\
\hline proportion of subjects not completing PC20 & & $423(82.8 \%)$ & $43(7.8 \%)$ \\
\hline proportion of subjects with current asthma ( & & $93(16.7 \%)$ & $0(0 \%)$ \\
\hline proportion of subjects with level-2 asthma ( & & $70(12.7 \%)$ & $0(0 \%)$ \\
\hline proportion of subjects with level-3 asthma ( & & $24(4.3 \%)$ & $0(0 \%)$ \\
\hline proportion of subjects with current wheeze & & $68(12.3 \%)$ & $0(0 \%)$ \\
\hline proportion of subjects with self-diagnosed ec & ma (SDE) & $146(26.3 \%)$ & $0(0 \%)$ \\
\hline proportion of subjects with doctor's diagnos & eczema (DDE) & $120(21.7 \%)$ & $0(0 \%)$ \\
\hline
\end{tabular}


Table 1 Study data (Continued)

\begin{tabular}{ccccccc}
\hline \hline & & cross-tabulation of clinical outcomes (\% of agreement) & & & \\
\hline & SDE & DDE & CA & A2 & CW & A3 \\
\hline SDE & & $95.47 \%$ & $74.82 \%$ & $74.82 \%$ & $73.37 \%$ & $74.09 \%$ \\
\hline DDE & $95.47 \%$ & & $77.17 \%$ & $77.90 \%$ & $76.09 \%$ & $78.26 \%$ \\
\hline CA & $74.82 \%$ & $77.17 \%$ & & $95.29 \%$ & $89.49 \%$ & $87.68 \%$ \\
\hline A2 & $74.82 \%$ & $77.90 \%$ & $95.29 \%$ & & $92.39 \%$ & $91.67 \%$ \\
\hline CW & $73.37 \%$ & $76.09 \%$ & $89.49 \%$ & $92.39 \%$ & $90.94 \%$ \\
\hline A3 & $74.09 \%$ & $78.26 \%$ & $87.68 \%$ & & $91.67 \%$ & $90.94 \%$ \\
\hline
\end{tabular}

Characteristics of the study population $(n=554)$ and cross-tabulation of outcomes.

(results were similar for the other outcomes and are available upon request). Different learning methods (LR, $\mathrm{RF}, \mathrm{AB}, \mathrm{DT}, \mathrm{OR})$ were applied to the full attribute set with the intent to identify both the best performing model (in terms of AUROC, sensitivity and specificity, given the high class imbalance of all outcomes) and to evaluate if non-linear models were able to improve the goodness-of-fit as compared to main-effects LR. RF consistently yielded the most predictive models (average AUROCs for CA, CW and DDE were $84 \%, 76 \%$, and $64 \%$, respectively), but the difference in mean AUROC with respect to $L R$ and $A B$ across 100 bootstrap runs could not be considered significantly shifted from zero at the 0.05 level. Instead, OR and DT were consistently superseded by RF. Figure 1 plots averaged ROC curves for the three different outcomes of CA, DDE and CW, whilst Table 2 shows averaged AUROC, and specificity at different sensitivity levels, both estimated from out-ofbag distributions across 100 bootstrap runs.

Based on these results, the RF method was retained and tested using different subsets of the original variable space - specifically the groups of allergen sensitisation, lung functions/airway hyper-responsiveness, demographic/environmental variables, and genetic variants (as defined in the methods) - in order to identify to which extent each group was contributing to increasing AUROC, sensitivity or specificity with respect to each outcome prediction. Table 3 shows averaged AUROC, and specificity at different sensitivity levels, both estimated from out-of-bag distributions across 100 bootstrap runs. For all outcomes, RF models using the allergen sensitisation yielded the best performance, followed in order by lung functions, demographic/environment, and genetic SNPs variable subsets. Usage of the whole variable set increased AUROC over each of the subsets for all outcomes (below the 0.05 significance level for demographic/environment and genetic feature subsets when predicting $\mathrm{CA}$ and $\mathrm{CW}$ ). Figure 2 shows receiver operating characteristic curves for the three outcomes considering each different feature subset, averaged across 100 bootstrap runs.

When looking at feature importance, the most relevant attributes reflected the overall performance of RF trained using feature subsets: variables from allergen sensitisation and lung function groups were the highest ranked, especially for CA and CW, as shown in Figure 3. Of note, when considering DDE, whole body impedance was the second most important variable reported by RF, and this was confirmed by LR, showing to a higher risk of DDE
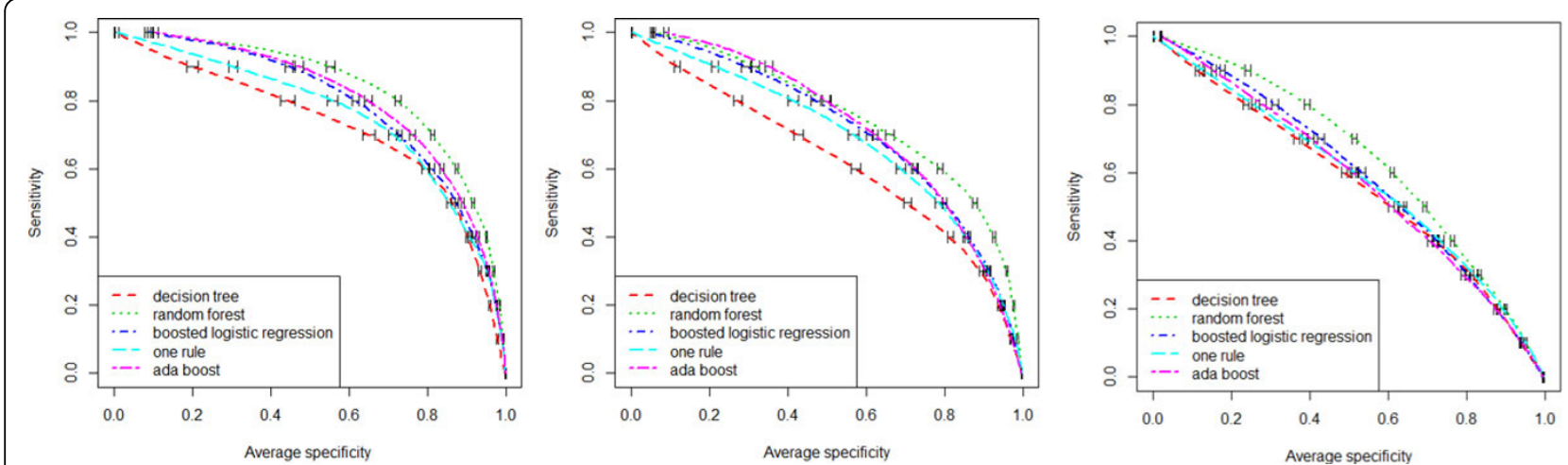

Figure 1 Comparison of machine learning methods. Performance comparison of different machine learning techniques in terms of area under the receiver operating characteristic curve in predicting current asthma (left panel), current wheeze (middle panel), and doctor's diagnosed eczema (right panel) using the whole feature set (demographic, environmental, genetic, lung function markers, and allergen sensitization). Results are out-of-bag predictions averaged over 100 bootstrap runs. 
Table 2 Comparison of machine learning methods.

\begin{tabular}{|c|c|c|c|c|c|}
\hline outcome & Model & AUROC & sensitivity (at $90 \%$ specificity) & sensitivity (at $\mathbf{8 0} \%$ specificity) & accuracy \\
\hline \multirow[t]{5}{*}{ Doctor's Diagnosed Eczema } & Decision Tree* & $0.57(0.04)$ & $0.15(0.07)$ & $0.29(0.07)$ & $0.78(0.02)$ \\
\hline & Random Forest & $0.64(0.03)$ & $0.2(0.06)$ & $0.34(0.07)$ & $0.79(0.02)$ \\
\hline & Logistic Regression & $0.59(0.04)$ & $0.18(0.06)$ & $0.31(0.08)$ & $0.78(0.02)$ \\
\hline & One Rule* & $0.58(0.06)$ & $0.2(0.11)$ & $0.3(0.15)$ & $0.79(0.02)$ \\
\hline & AdaBoost & $0.58(0.04)$ & $0.17(0.06)$ & $0.3(0.07)$ & $0.78(0.02)$ \\
\hline \multirow[t]{5}{*}{ Current Asthma } & Decision Tree* & $0.72(0.06)$ & $0.39(0.12)$ & $0.54(0.11)$ & $0.85(0.02)$ \\
\hline & Random Forest & $0.84(0.03)$ & $0.55(0.09)$ & $0.72(0.08)$ & $0.87(0.02)$ \\
\hline & Logistic Regression & $0.79(0.04)$ & $0.45(0.08)$ & $0.63(0.08)$ & $0.86(0.02)$ \\
\hline & One Rule* & $0.76(0.06)$ & $0.44(0.09)$ & $0.61(0.11)$ & $0.86(0.02)$ \\
\hline & AdaBoost & $0.81(0.04)$ & $0.48(0.09)$ & $0.66(0.07)$ & $0.86(0.02)$ \\
\hline \multirow[t]{5}{*}{ Current Wheeze } & Decision Tree* & $0.62(0.06)$ & $0.27(0.1)$ & $0.36(0.11)$ & $0.88(0.02)$ \\
\hline & Random Forest & $0.76(0.04)$ & $0.47(0.09)$ & $0.6(0.09)$ & $0.89(0.02)$ \\
\hline & Logistic Regression & $0.72(0.04)$ & $0.34(0.08)$ & $0.51(0.08)$ & $0.88(0.02)$ \\
\hline & One Rule* & $0.69(0.06)$ & $0.33(0.09)$ & $0.49(0.12)$ & $0.88(0.02)$ \\
\hline & AdaBoost & $0.73(0.04)$ & $0.32(0.09)$ & $0.5(0.09)$ & $0.88(0.02)$ \\
\hline
\end{tabular}

Performance of machine learning models on different outcomes using the full set of demographic, environmental, genetic (single nucleotide polymorphisms), allergen sensitisation, and lung functions variables. Results are mean (standard deviation) values estimated from out-of-bag distributions across 100 bootstrap runs. * difference in AUROC significantly shifted from zero at the 0.05 level as compared to that of a random forest. AUROC: area under the receiver operating characteristic curve.

(OR 1.19 per square root increase, 95\% CI 1.13-1.27, $\mathrm{p}$-value $=0.0016)$, and found to be correlated to BMI (Pearson's $\rho=-0.54$, $\mathrm{p}$-value $<0.0001$ ). No SNPs were scored within the $25^{\text {th }}$ percentile of RF importance, but crude associations with asthma symptoms were confirmed. When considering CW, rs4986790 was the SNP with highest level of association using a chi-square test on allele categories (unadjusted $\mathrm{p}=0.005$ ), whilst rs6037651 using the additive model (unadjusted $\mathrm{p}=0.003$ ). When considering DDE, rs2569190 (unadjusted $\mathrm{p}=0.003$ from chisquare) and rs574174 (unadjusted $\mathrm{p}=0.004$ from additive model). However their significance was not below the 0.05 level when correcting for multiple testing (using Benjamini-Hochberg correction). For CA, rs7212938 and rs8079416 were the top-scoring SNPs under the categorical and additive model, respectively (unadjusted $\mathrm{p}=$ 0.0002 and $p=0.0006$ ), and the significance remained below the 0.1 level after adjusting for multiple testing ( $\mathrm{p}=$ 0.09 and $\mathrm{p}=0.03$ ).

To compare more thoroughly RF with LR, we analysed the variable sets selected by the LogitBoost algorithm. Specifically, for CW, five predictors were selected: IgE of house dust mite $\left(\mathrm{OR}=1.207\right.$ per $\log _{\mathrm{e}}$ higher, $\left.\mathrm{p}=0.005\right)$; IgE of $\operatorname{dog}\left(\mathrm{OR}=1.465\right.$ per $\log _{\mathrm{e}}$ higher, $\left.\mathrm{p}<0.0001\right)$; number of cigarettes smoked $(\mathrm{OR}=1.032$ per packages/year, $\mathrm{p}=0.003)$; moving house $(\mathrm{OR}=3.078$ for moving twice or more as compared to not moving, $\mathrm{p}=0.001$ ); MDRS $(\mathrm{OR}=0.794$ per transformed unit $\mathrm{p}=0.0004)$. For $\mathrm{CA}$, nine predictors were selected: IgE of house dust mite $\left(\mathrm{OR}=1.308\right.$ per $\log _{\mathrm{e}}$ higher, $\left.\mathrm{p}<0.0001\right)$; IgE of dog $(\mathrm{OR}$
$=1.519$ per $\log _{\mathrm{e}}$ higher, $\left.\mathrm{p}<0.0001\right)$; job causing wheezing problems $(\mathrm{OR}=13.923$ for presence of condition; $\mathrm{p}<$ $0.0001)$; rs8079416 (OR $=0.502$ as additive model; $\mathrm{p}=0.002) ; \mathrm{rs} 11540720(\mathrm{OR}=0.182$ as additive model; $\mathrm{p}=0.008)$; $\mathrm{rs} 5743704$ (OR $=0.265$ as additive model; $\mathrm{p}=$ $0.011)$; $r$ 11536889 $(\mathrm{OR}=0.265$ as additive model; $\mathrm{p}=$ $0.011)$; sRaw $\left(\mathrm{OR}=6.509\right.$ per $\log _{\mathrm{e}}$ higher; $\left.\mathrm{p}=0.0009\right)$; MDRS (OR $=0.839$ per transformed unit $\mathrm{p}=0.013$ ). For $\mathrm{DDE}$, one predictor was selected, the IgE of cat $(\mathrm{OR}=$ 1.378 per $\log _{\mathrm{e}}$ higher, $\left.\mathrm{p}<0.0001\right)$. All features selected by LogitBoost were listed as top-ranked variables by the RF, except for SNPs in the CA outcome. Note that these LR models were obtained from one data set using a single LogitBoost selection, and - given also the degree of correlation among variables - alternative models with equal performance may be selected by varying selection heuristics.

\section{Discussion}

We investigated the ability of linear and non-linear machine learning models to predict asthma, wheezing, and eczema outcomes, according to different operational definitions, with a heterogeneous set of attributes in an adult population. Models were compared in terms of performance, complexity and interpretability. Different feature groups were evaluated and combined in order to understand determinants (and combinations thereof) of asthma symptoms or the presence of eczema. The use of random forests in model building yielded better AUROC, sensitivity and specificity than other methods. This might 
Table 3 Comparison of random forest performance using selected input domains.

\begin{tabular}{|c|c|c|c|c|c|c|}
\hline outcome & feature set & AUROC & $p$-value* & $\begin{array}{l}\text { sensitivity (at 90\% } \\
\text { specificity) }\end{array}$ & $\begin{array}{l}\text { sensitivity (at } 80 \% \\
\text { specificity) }\end{array}$ & accuracy \\
\hline \multirow[t]{5}{*}{$\begin{array}{l}\text { Doctor's Diagnosed } \\
\text { Eczema }\end{array}$} & allergens & $\begin{array}{l}0.62 \\
(0.03)\end{array}$ & 0.34 & $0.22(0.06)$ & $0.37(0.06)$ & $\begin{array}{l}0.79 \\
(0.02)\end{array}$ \\
\hline & lung functions & $\begin{array}{l}0.56 \\
(0.04)\end{array}$ & 0.08 & $0.13(0.05)$ & $0.24(0.06)$ & $\begin{array}{l}0.78 \\
(0.02)\end{array}$ \\
\hline & genetic & $\begin{array}{l}0.56 \\
(0.04) \\
\end{array}$ & 0.11 & $0.14(0.05)$ & $0.25(0.06)$ & $\begin{array}{l}0.78 \\
(0.02) \\
\end{array}$ \\
\hline & $\begin{array}{l}\text { demographic/ } \\
\text { environ. }\end{array}$ & $\begin{array}{l}0.56 \\
(0.04)\end{array}$ & 0.05 & $0.12(0.05)$ & $0.24(0.07)$ & $\begin{array}{l}0.78 \\
(0.02)\end{array}$ \\
\hline & all & $\begin{array}{l}0.65 \\
(0.04)\end{array}$ & reference & $0.2(0.07)$ & $0.35(0.08)$ & $\begin{array}{l}0.79 \\
(0.02)\end{array}$ \\
\hline \multirow[t]{5}{*}{ Current Asthma } & allergens & $\begin{array}{l}0.79 \\
(0.04)\end{array}$ & 0.11 & $0.43(0.08)$ & $0.64(0.07)$ & $\begin{array}{l}0.86 \\
(0.02)\end{array}$ \\
\hline & lung functions & $\begin{array}{l}0.76 \\
(0.04)\end{array}$ & 0.04 & $0.44(0.08)$ & $0.6(0.09)$ & $\begin{array}{l}0.86 \\
(0.02)\end{array}$ \\
\hline & genetic & $\begin{array}{l}0.54 \\
(0.04) \\
\end{array}$ & $<0.0001$ & $0.12(0.05)$ & $0.23(0.07)$ & $\begin{array}{l}0.83 \\
(0.02) \\
\end{array}$ \\
\hline & $\begin{array}{l}\text { demographic/ } \\
\text { environ. }\end{array}$ & $\begin{array}{l}0.62 \\
(0.04) \\
\end{array}$ & $<0.0001$ & $0.2(0.08)$ & $0.38(0.07)$ & $\begin{array}{l}0.83 \\
(0.02) \\
\end{array}$ \\
\hline & all & $\begin{array}{l}0.84 \\
(0.03) \\
\end{array}$ & reference & $0.56(0.09)$ & $0.73(0.08)$ & $\begin{array}{l}0.87 \\
(0.02) \\
\end{array}$ \\
\hline \multirow[t]{5}{*}{ Current Wheeze } & allergens & $\begin{array}{l}0.75 \\
(0.04) \\
\end{array}$ & 0.35 & $0.34(0.09)$ & $0.54(0.1)$ & $\begin{array}{l}0.88 \\
(0.02) \\
\end{array}$ \\
\hline & lung functions & $\begin{array}{l}0.72 \\
(0.05)\end{array}$ & 0.19 & $0.42(0.09)$ & $0.55(0.08)$ & $\begin{array}{l}0.89 \\
(0.02)\end{array}$ \\
\hline & genetic & $0.5(0.05)$ & 0.0002 & $0.11(0.06)$ & $0.21(0.08)$ & $\begin{array}{l}0.88 \\
(0.02)\end{array}$ \\
\hline & $\begin{array}{l}\text { demographic/ } \\
\text { environ. }\end{array}$ & $0.6(0.05)$ & 0.006 & $0.17(0.07)$ & $0.32(0.09)$ & $\begin{array}{l}0.88 \\
(0.02) \\
\end{array}$ \\
\hline & all & $\begin{array}{l}0.77 \\
(0.04)\end{array}$ & reference & $0.5(0.09)$ & $0.62(0.07)$ & $\begin{array}{l}0.89 \\
(0.02)\end{array}$ \\
\hline
\end{tabular}

Performance of random forest on different outcomes using specific variable subsets and the full set of demographic, environmental, genetic (single nucleotide polymorphisms), allergen sensitisation, and lung functions variables. Results are mean (standard deviation) estimated from out-of-bag distributions across 100 bootstrap runs.

"testing the hypothesis of difference in AUROC significantly shifted from zero as compared to that of a random forest model using all variables with a corrected paired t-test.

AUROC: area under the receiver operating characteristic curve.
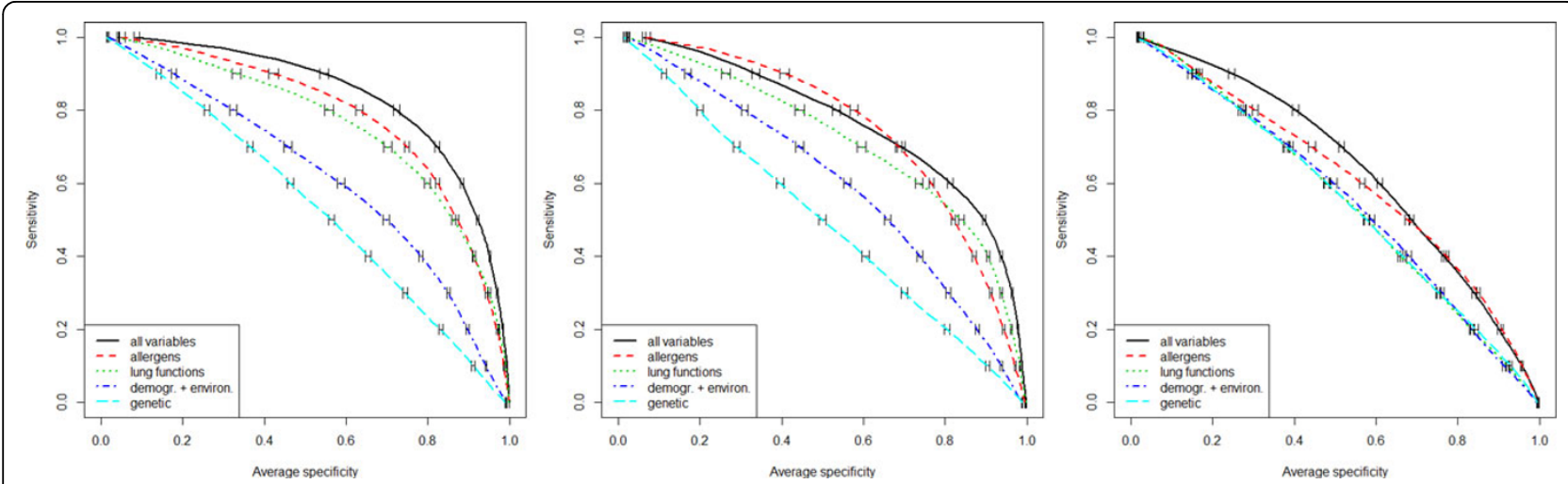

Figure 2 Comparison of random forest performance using selected input domains. Performance comparison of random forests in terms of area under the receiver operating characteristic curve in predicting current asthma (left panel), current wheeze (middle panel), and doctor's diagnosed eczema (right panel) using the whole feature set (demographic, environmental, genetic, lung function markers, and allergen sensitization) and selected feature subsets. Results are out-of-bag predictions averaged over 100 bootstrap runs. 

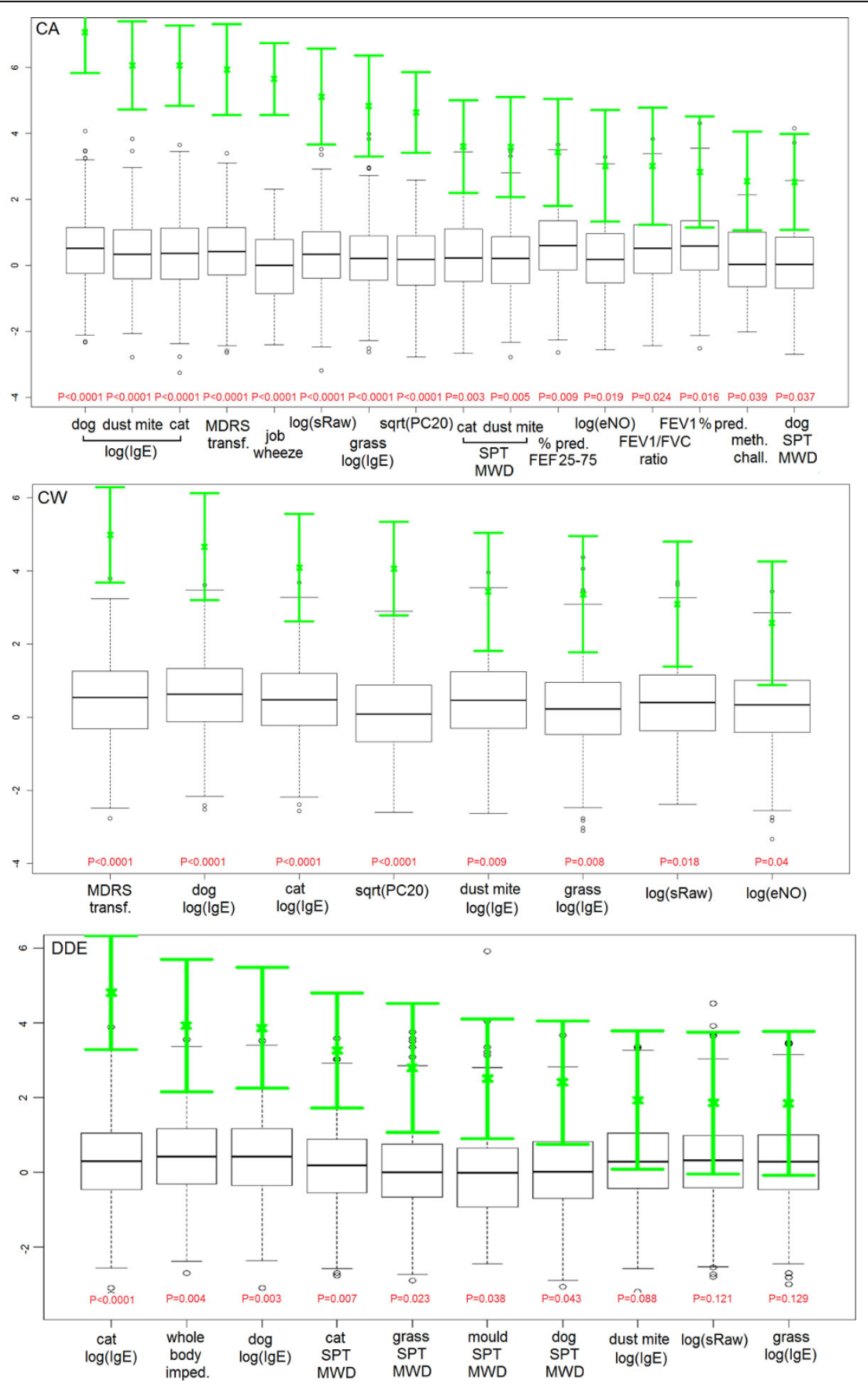

Figure 3 Feature importance evaluation by means of random forests. Importance is calculated and shown as the rescaled mean (standard deviation) decrease in accuracy over 1000 independent runs (green colour). Boxplots represent a null feature importance distribution obtained by permuting the outcome randomly for 1000 times. Variables significant at the 0.1 level ( $p$-values in red) are shown for current asthma (upper panel), current wheeze (middle panel), and doctor's diagnosed eczema (lower panel) using the whole feature set as input.

be due to the ability of random forests to model non-linear functions and account for variable interactions, although the corrected t-test on AUROC did not show a statistically significant difference. Furthermore, the difference between random forests and logistic regression was minimal in predicting asthma phenotypes. In terms of statistical power, however, a larger sample of subjects may reveal undetected differences in the AUROC comparisons of the linear and non-linear methods.

There was a higher prevalence of eczema compared to asthma and wheeze in the population; AUROCs were higher when considering current asthma and current 
wheeze outcomes (0.84 and 0.76), lower for doctor's diagnosed eczema (0.64). Results show clearly that there is a benefit of merging information from different sources, e.g. lung functions, allergen sensitization tests, genetic markers, demographics and environment. However, in general all models were characterized by a relatively low sensitivity with any feature set combination. A lower sensitivity was obtained compared to that of Chatzimichail et al.[23], who used a similar outcome definition: this is because we explicitly excluded any previous personal and familiar diagnosis of asthma, wheeze and eczema from the input set, given the fact that outcomes are often defined recursively on previous episodes. In fact, when utilising previous diagnoses (plus anti-asthma medication usage), sensitivity increased to $\geq 0.8$ ( $\geq 0.9$ when including anti-asthma medication usage variables) at a minimum specificity of 0.9 . However, direct comparison with other methods is only qualitative given the different study designs and populations.

Regarding the importance of features, our findings confirm the important contribution of allergen sensitization (dust mite, dog, cat), along with lung function markers, in predicting asthma diagnoses or symptom patterns. The predictive ability of genetic markers alone is limited, although for the current asthma outcome the LogitBoost algorithm selected a few over the whole set of variables. Our AUROCs for SNPs are in line with the previous estimates of Spycher et al.[20], who analysed the genome-wide prediction of childhood asthma and related phenotypes in a longitudinal birth cohort (reporting AUROC of 0.59 for wheeze and of 0.54 for asthma). However, our analysis was not focused on genetic markers: a limited population sample, in terms of the set candidate SNPs as well as of environmental markers, can decrease the power to look for SNP-environment interactions effectively; therefore a more accurate study design is warranted for this objective.

We observed interesting novel and biologically plausible association between bio-impedance and eczema. Previous studies have found that whole body impedance is associated with steroid treatments[45] and several types of cutaneous reactions[46], including an indirect association to Filaggrin-related eczema (via stratum corneum hydration) [47]. Further investigation of this association is warranted.

Limitation of our study include the use of an in-house, rather than externally validated assay for component resolved diagnostics (however, this metric was coupled with validated skin prick testing and blood Immunoglobulin E testing), and the facts that genetic analysis was restricted to candidate genes. Another potential limitation was the naive policy for missing value imputation; however the extent of missing information was negligible.

\section{Conclusions}

Being a cross-sectional study, with no longitudinal separation of predictors and outcomes, this study is not intended to assess different approaches to causal inference. However, our data demonstrate that even with cross-sectional data, there is considerable scope to build more usefully complex models to better understand asthma and other complex diseases (such as eczema). Future studies might incorporate more factors/attributes and harness longitudinal data in the prediction of later clinical outcomes.

\section{Competing interests}

The authors have no competing interests to declare in relation to this manuscript.

\section{Authors' contributions}

MCFP manuscript writing, machine learning analysis; SM genetic data preprocessing, data collection; AS study design, data collection; AC study design, data management; IEB statistical review. All authors reviewed and contributed to specific sections of the manuscript.

\section{Declarations}

Publication for this article has been funded by grants from J P Moulton Charitable Foundation (sponsoring the MAAS cohort), Medical Research Council (MRC) grants G0601361, MR/K002449/1, University of Manchester's Library via the Research Councils UK (for open-access publications), and by the MRC Health eResearch Centre (HeRC) grant MR/K006665/1.

This article has been published as part of BMC Medical Genomics Volume 7 Supplement 1, 2014: Selected articles from the 3rd Translational Bioinformatics Conference (TBC/ISCB-Asia 2013). The full contents of the supplement are available online at http://www.biomedcentral.com/ bmcmedgenomics/supplements/7/S1.

\section{Authors' details}

${ }^{1}$ Centre for Health Informatics, Institute of Population Health, Faculty of Medical and Human Sciences, University of Manchester, Manchester, UK. ${ }^{2}$ Centre for Respiratory Medicine and Allergy, Institute of Inflammation and Repair, University of Manchester, Manchester, UK.

Published: 8 May 2014

\section{References}

1. Papierniak ES, Lowenthal DT, Harman E: Novel therapies in asthma: leukotriene antagonists, biologic agents, and beyond. Am J Ther 2013, 20(1):79-103.

2. Bacharier $L B$, Guilbert TW: Diagnosis and management of early asthma in preschool-aged children. J Allergy Clin Immunol 2012, 130(2):287-296, quiz 297-288.

3. Lotvall J, Akdis CA, Bacharier LB, Bjermer L, Casale TB, Custovic A, Lemanske RF, Wardlaw AJ, Wenzel SE, Greenberger PA: Asthma endotypes: a new approach to classification of disease entities within the asthma syndrome. J Allergy Clin Immunol 2011, 127(2):355-360.

4. Sittka A, Vera J, Lai X, Schmeck B: Asthma phenotyping, therapy, and prevention: what can we learn from systems biology? Pediatr Res 2013.

5. Taylor PE, Jacobson KW, House JM, Glovsky MM: Links between pollen, atopy and the asthma epidemic. International archives of allergy and immunology 2007, 144(2):162-170.

6. Gent JF, Belanger K, Triche EW, Bracken MB, Beckett WS, Leaderer BP: Association of pediatric asthma severity with exposure to common household dust allergens. Environmental research 2009, 109(6):768-774.

7. Wang J, Calatroni A, Visness CM, Sampson HA: Correlation of specific lgE to shrimp with cockroach and dust mite exposure and sensitization in an inner-city population. J Allergy Clin Immunol 2011, 128(4):834-837.

8. Sordillo JE, Webb T, Kwan D, Kamel J, Hoffman E, Milton DK, Gold DR: Allergen exposure modifies the relation of sensitization to fraction of exhaled nitric oxide levels in children at risk for allergy and asthma. J Allergy Clin Immunol 2011, 127(5):1165-1172 e1165.

9. Burrows B, Martinez FD, Halonen M, Barbee RA, Cline MG: Association of asthma with serum IgE levels and skin-test reactivity to allergens. $N$ Engl J Med 1989, 320(5):271-277. 
10. Beeh KM, Ksoll M, Buhl R: Elevation of total serum immunoglobulin $E$ is associated with asthma in nonallergic individuals. The European respiratory journal : official journal of the European Society for Clinical Respiratory Physiology 2000, 16(4):609-614.

11. Simpson BM, Custovic A, Simpson A, Hallam CL, Walsh D, Marolia $H$, Campbell J, Woodcock A: NAC Manchester Asthma and Allergy Study (NACMAAS): risk factors for asthma and allergic disorders in adults. Clinical and experimental allergy : journal of the British Society for Allergy and Clinical Immunology 2001, 31(3):391-399.

12. Marinho S, Simpson A, Soderstrom L, Woodcock A, Ahlstedt S, Custovic A: Quantification of atopy and the probability of rhinitis in preschool children: a population-based birth cohort study. Allergy 2007, 62(12):1379-1386.

13. Marinho S, Simpson A, Marsden P, Smith JA, Custovic A: Quantification of atopy, lung function and airway hypersensitivity in adults. Clinical and translational allergy 2011, 1(1):16.

14. Castro-Rodriguez JA, Holberg CJ, Wright AL, Martinez FD: A clinical index to define risk of asthma in young children with recurrent wheezing. Am J Respir Crit Care Med 2000, 162(4 Pt 1):1403-1406.

15. Singer F, Luchsinger I, Inci D, Knauer N, Latzin P, Wildhaber JH, Moeller A: Exhaled nitric oxide in symptomatic children at preschool age predicts later asthma. Allergy 2013, 68(4):531-538.

16. Greenberg S: Asthma exacerbations: predisposing factors and prediction rules. Current opinion in allergy and clinical immunology 2013.

17. Wadsworth SJ, Sandford AJ: Personalised medicine and asthma diagnostics/management. Current allergy and asthma reports 2013, 13(1):118-129.

18. Pralong JA, Seed MJ, Yasri R, Agius RM, Cartier A, Labrecque M: A computer based asthma hazard prediction model and new molecular weight agents in occupational asthma. Occupational and environmental medicine 2013, 70(1):70.

19. Soyiri IN, Reidpath DD: Semistructured black-box prediction: proposed approach for asthma admissions in London. International journal of general medicine 2012, 5:693-705.

20. Spycher BD, Henderson J, Granell R, Evans DM, Smith GD, Timpson NJ, Sterne JA: Genome-wide prediction of childhood asthma and related phenotypes in a longitudinal birth cohort. J Allergy Clin Immunol 2012, 130(2):503-509 e507.

21. Savenije OE, Kerkhof M, Koppelman GH, Postma DS: Predicting who will have asthma at school age among preschool children. J Allergy Clin Immunol 2012, 130(2):325-331.

22. Vial Dupuy A, Amat F, Pereira B, Labbe A, Just J: A simple tool to identify infants at high risk of mild to severe childhood asthma: the persistent asthma predictive score. The Journal of asthma: official journal of the Association for the Care of Asthma 2011, 48(10):1015-1021.

23. Chatzimichail E, Paraskakis E, Sitzimi M, Rigas A: An intelligent system approach for asthma prediction in symptomatic preschool children. Computational and mathematical methods in medicine 2013, 2013:240182.

24. Marinho S, Custovic A, Marsden P, Smith JA, Simpson A: 17q12-21 variants are associated with asthma and interact with active smoking in an adult population from the United Kingdom. Annals of allergy, asthma \& immunology : official publication of the American College of Allergy, Asthma, \& Immunology 2012, 108(6):402-411 e409.

25. Custovic A, Simpson BM, Murray CS, Lowe L, Woodcock A, Asthma NACM Allergy Study G: The National Asthma Campaign Manchester Asthma and Allergy Study. Pediatric allergy and immunology : official publication of the European Society of Pediatric Allergy and Immunology 2002, 13(Suppl 15):32-37.

26. Langley SJ, Goldthorpe S, Craven M, Morris J, Woodcock A, Custovic A: Exposure and sensitization to indoor allergens: association with lung function, bronchial reactivity, and exhaled nitric oxide measures in asthma. J Allergy Clin Immunol 2003, 112(2):362-368.

27. Langley SJ, Goldthorpe S, Custovic A, Woodcock A: Relationship among pulmonary function, bronchial reactivity, and exhaled nitric oxide in a large group of asthmatic patients. Annals of allergy, asthma \& immunology : official publication of the American College of Allergy, Asthma, \& Immunology 2003, 91(4):398-404.

28. Kidon MI, Chiang WC, Liew WK, Ong TC, Tiong YS, Wong KN, Angus AC, Ong ST, Gao YF, Reginald K, et al: Mite component-specific IgE repertoire and phenotypes of allergic disease in childhood: the tropical perspective. Pediatric allergy and immunology : official publication of the European Society of Pediatric Allergy and Immunology 2011, 22(2):202-210.

29. de Marco R, Marcon A, Jarvis D, Accordini S, Almar E, Bugiani M, Carolei A, Cazzoletti L, Corsico A, Gislason D, et al: Prognostic factors of asthma severity: a 9-year international prospective cohort study. J Allergy Clin Immunol 2006, 117(6):1249-1256.

30. Siroux V, Boudier A, Anto JM, Cazzoletti L, Accordini S, Alonso J, Cerveri I, Corsico A, Gulsvik A, Jarvis D, et al: Quality-of-life and asthma-severity in general population asthmatics: results of the ECRHS II study. Allergy 2008, 63(5):547-554.

31. Pekkanen J, Sunyer J, Anto JM, Burney P, European Community Respiratory Health S: Operational definitions of asthma in studies on its aetiology. The European respiratory journal : official journal of the European Society for Clinical Respiratory Physiology 2005, 26(1):28-35.

32. Barrett JC, Fry B, Maller J, Daly MJ: Haploview: analysis and visualization of LD and haplotype maps. Bioinformatics 2005, 21(2):263-265.

33. Gabriel SB, Schaffner SF, Nguyen H, Moore JM, Roy J, Blumenstiel B, Higgins J, DeFelice M, Lochner A, Faggart M, et al: The structure of haplotype blocks in the human genome. Science 2002, 296(5576):2225-2229.

34. Landwehr N, Hall M, Frank E: Logistic model trees. Mach Learn 2005, 59(1-2):161-205.

35. Venables WN, Ripley BD: Modern Applied Statistics with S Springer; 2002.

36. Breiman L, Friedman J, Stone C, Olshen RA: Classification and Regression Trees. Chapman and Hall/CRC; 1984.

37. Breiman L: Random forests. Mach Learn 2001, 45(1):5-32.

38. Freund $Y$, Schapire RE: A decision-theoretic generalization of on-line learning and an application to boosting. J Comput Syst Sci 1997, 55(1):119-139.

39. Hastie T, Tibshirani R, Friedman JH: The elements of statistical learning : data mining, inference, and prediction. New York, NY: Springer; 22009.

40. Nadeau C, Bengio Y: Inference for the Generalization Error. Mach Learn 2003, 52(3):239-281.

41. Garcia S, Herrera F: An Extension on "Statistical Comparisons of Classifiers over Multiple Data Sets" for all Pairwise Comparisons. J Mach Learn Res 2008, 9:2677-2694.

42. Altmann A, Tolosi L, Sander O, Lengauer T: Permutation importance: a corrected feature importance measure. Bioinformatics 2010, 26(10):1340-1347.

43. Nicodemus KK, Malley JD, Strobl C, Ziegler A: The behaviour of random forest permutation-based variable importance measures under predictor correlation. BMC Bioinformatics 2010, 11:110

44. Strobl C, Boulesteix AL, Zeileis A, Hothorn T: Bias in random forest variable importance measures: illustrations, sources and a solution. $B M C$ Bioinformatics 2007, 8:25.

45. Heitmann BL, Anhoj J, Bisgaard AM, Ward L, Bisgaard H: Changes in body water distribution during treatment with inhaled steroid in pre-school children. Annals of human biology 2004, 31(3):333-341.

46. Nyren $M$, Hagstromer L, Emtestam L: On assessment of skin reactivity using electrical impedance. Ann Ny Acad Sci 1999, 873:214-220.

47. Nemoto-Hasebe I, Akiyama M, Nomura T, Sandilands A, McLean WHI, Shimizu H: Clinical Severity Correlates with Impaired Barrier in FilaggrinRelated Eczema. J Invest Dermatol 2009, 129(3):682-689.

doi:10.1186/1755-8794-7-S1-S7

Cite this article as: Prosperi et al:: Predicting phenotypes of asthma and eczema with machine learning. BMC Medical Genomics 2014 7(Suppl 1):S7. 